\title{
Tracking of Noise Tolerance to Measure Hearing Aid Benefit
}

DOI: $10.3766 /$ jaaa. 16053

\author{
Francis Kuk* \\ Eric Seper* \\ Chi-Chuen Lau* \\ Petri Korhonen*
}

\begin{abstract}
Background: The benefits offered by noise reduction (NR) features on a hearing aid had been studied traditionally using test conditions that set the hearing aids into a stable state of performance. While adequate, this approach does not allow the differentiation of two NR algorithms that differ in their timing characteristics (i.e., activation and stabilization time).

Purpose: The current study investigated a new method of measuring noise tolerance (Tracking of Noise Tolerance [TNT]) as a means to differentiate hearing aid technologies. The study determined the withinsession and between-session reliability of the procedure. The benefits provided by various hearing aid conditions (aided, two NR algorithms, and a directional microphone algorithm) were measured using this procedure. Performance on normal-hearing listeners was also measured for referencing.
\end{abstract}

Research Design: A single-blinded, repeated-measures design was used.

Study Sample: Thirteen experienced hearing aid wearers with a bilaterally symmetrical ( $\leq 10 \mathrm{~dB})$ mild-to-moderate sensorineural hearing loss participated in the study. In addition, seven normal-hearing listeners were tested in the unaided condition.

Data Collection and Analysis: Participants tracked the noise level that met the criterion of tolerable noise level (TNL) in the presence of an $85 \mathrm{~dB}$ SPL continuous discourse passage. The test conditions included an unaided condition and an aided condition with combinations of NR and microphone modes within the UNIQUE hearing aid (omnidirectional microphone, no NR; omnidirectional microphone, NR; directional microphone, no NR; and directional microphone, NR) and the DREAM hearing aid (omnidirectional microphone, no NR; omnidirectional microphone, NR). Each tracking trial lasted 2 min for each hearing aid condition. Normal-hearing listeners tracked in the unaided condition only. Nine of the 13 hearingimpaired listeners returned after 3 mo for retesting in the unaided and aided conditions with the UNIQUE hearing aid. The individual TNL was estimated for each participant for all test conditions. The TNT index was calculated as the difference between $85 \mathrm{~dB}$ SPL and the TNL.

Results: The TNT index varied from $2.2 \mathrm{~dB}$ in the omnidirectional microphone, no NR condition to $-4.4 \mathrm{~dB}$ in the directional microphone, NR on condition. Normal-hearing listeners reported a TNT index of $-5.7 \mathrm{~dB}$ using this procedure. The averaged improvement in TNT offered by the NR algorithm on the UNIQUE varied from $2.1 \mathrm{~dB}$ when used with a directional microphone to $3.0 \mathrm{~dB}$ when used with the omnidirectional microphone. The time course of the NR algorithm was different between the UNIQUE and the DREAM hearing aids, with the UNIQUE reaching a stable TNL sooner than the DREAM. The averaged improvement in TNT index from the UNIQUE directional microphone was $3.6 \mathrm{~dB}$ when NR was activated and $4.4 \mathrm{~dB}$ when NR was deactivated. Together, directional microphone and NR resulted in a total TNT improvement of $6.5 \mathrm{~dB}$. The test-retest reliability of the procedure was high, with an intrasession $95 \%$ confidence interval $(\mathrm{Cl})$ of $2.2 \mathrm{~dB}$ and an intersession $95 \% \mathrm{Cl}$ of $4.2 \mathrm{~dB}$.

Conclusions: The effect of the NR and directional microphone algorithms was measured to be 2-3 and 3.6-4.4 dB, respectively, using the TNT procedure. Because of its tracking property and reliability, this procedure may hold promise in differentiating among some hearing aid features that also differ in their time course of action.

*Widex Office of Research in Clinical Amplification (ORCA-USA), Lisle, IL

Corresponding author: Francis Kuk, Widex Office of Research in Clinical Amplification (ORCA-USA), Lisle, IL 60532; E-mail: fkuk@widex.com 
Key Words: directional microphone, noise reduction, reliability, tolerable noise level, tracking noise tolerance

\begin{abstract}
Abbreviations: $\mathrm{ANL}=$ Acceptable Noise Level; $\mathrm{Cl}=$ confidence interval; $\mathrm{CST}=$ Connected Speech Test; dir = directional; NR = noise reduction; omni = omnidirectional; RIC = receiver-in-the-canal; rtSE = Real-Time Speech Enhancer; SD = standard deviation; SE = Speech Enhancer; se = standard error; SII = speech intelligibility index; SNR = signal-to-noise ratio; TNL = tolerable noise level; TNT = Tracking of Noise Tolerance
\end{abstract}

\section{INTRODUCTION}

$\mathrm{N}$ oise reduction (NR) algorithms have been available in hearing aids for many years. These algorithms have demonstrated improvements in speech intelligibility (Peeters et al, 2009), sound quality (Ricketts and Hornsby, 2005), learning of novel speech (Marcoux et al, 2006), recall (Ng et al, 2015), listening comfort (Desjardins and Doherty, 2014), and noise tolerance (Lowery and Plyler, 2013; Wu and Stangl, 2013). Understandably, these evaluations assessed the performance of the NR algorithms when they were fully activated and stabilized (i.e., at a steady state). This study reported on the preliminary findings of using a tool that allowed a comparison of NR algorithms with different stabilization times on a speech-in-noise task.

NR algorithms operate by reducing gain in frequency channels where "nonspeech" signals are identified (Chung, 2004). How quickly the "speech" and "nonspeech" distinction is made and how quickly the algorithm reduces gain vary across commercial systems (Chung, 2004). Such differences in system responsiveness could affect how quickly the hearing aid wearers realize the benefit of the NR algorithm upon entering a new noisy environment. A faster system (shorter activation/stabilization time) could ensure relatively easy listening within a short time; however, such a system could introduce artifacts like musical noise. A longer activation time would have minimized many artifacts; however, it may take some time (maybe 20-30 sec) for the wearers to fully realize such an action. This could ultimately affect the wearer's satisfaction with the hearing aid.

One of the new features in the Widex (Lynge, Denmark) UNIQUE hearing aid is an NR algorithm called Real-Time Speech Enhancer (rtSE). This is an updated version of the Speech Enhancer (SE) feature that was introduced with the Inteo (and continued into the DREAM) hearing aid (Kuk and Paludan-Muller, 2006). This algorithm attempts to maximize the speech intelligibility index (SII) in a noisy situation. In its original version, the system first identifies the listening situation as "speech" versus "nonspeech" (or noise). The detection of "noise" activates the SE feature. The algorithm samples the spectrum and level of the background noise, estimates the speech spectrum, and then adjusts gain in all 15 channels so that the speech spectrum is above the hearing loss and the noise spectrum and that the estimated
SII is maximized without loudness discomfort. The amount of gain reduction depends on the noise level, signal-to-noise ratio (SNR) of the environment, and the degree of hearing loss of the wearer. A maximum gain reduction of $12 \mathrm{~dB}$ is allowed. When appropriate (no feedback and when SII can be improved), gain increase of up to $4 \mathrm{~dB}$ in the mid frequencies is allowed to further enhance the estimated SII. Peeters et al (2009) showed that this algorithm enhanced the SNR measured on the Hearing in Noise Test (Nilsson et al, 1994) and the Acceptable Noise Level (ANL; Nabelek et al, 1991) test by $3 \mathrm{~dB}$ when used with an omnidirectional microphone.

The rtSE in the UNIQUE hearing aid is fundamentally the same as the SE in the Inteo/DREAM except for a faster activation time. The DREAM SE system takes $15-20$ sec to activate and $\sim 30$ sec to reach a final state of gain reduction. In contrast, the UNIQUE rtSE takes only 2-3 sec to activate and $<5$ sec to reach a final gain state. A shorter stabilization time means the system can respond to the changes in the environment sooner, possibly resulting in more consistent speech intelligibility and listening comfort than one that takes a longer stabilization time. This subtle difference between the two versions of the SE may not be distinguishable using the traditional procedures for evaluating NR algorithms because they measure performance when the algorithm has reached a stable state. A tool that demonstrates timing differences between two NR algorithms would be desirable. Such a tool must be sensitive to the differences in timing and be reliable enough so that the measured difference is greater than the known variability in listener responses.

One approach to allow examination of the timing difference between NR algorithms is to have listeners track their loudness perception over time. If one assumes in most speech-in-noise situations that the talker speaks at a constant level, and all NR algorithms attempt to lower the impact of surrounding "noise," it is reasonable to fix the speech level and vary the noise level to reach the specified criterion. While the noise is presented, listeners will be asked to adjust the level of the background noise so it meets a specific criterion (e.g., "putting up" with the noise while following the speech). The duration of the tracking must be fixed; it cannot be too long, to minimize the risk of fatigue, but long enough to reach a stable judgment. Sampling of the listeners' perceived loudness should be performed frequently so that information on the changes 
in loudness perception over the noise sample is available. The estimate of the tolerable noise level (TNL) should be taken as the average level where the loudness perception is stable. Noise tracking over a fixed duration could also overcome the variability in TNL associated with normal fluctuations in loudness judgment and the uneven time spent among listeners when measuring noise tolerance. Another advantage of a tracking procedure is that it also provides loudness information over time. Someone who can tolerate less noise as time progresses or those who report lower loudness over time (e.g., decruitment) may prompt additional investigation. The effects of signal processing algorithms that differ in their time courses may be revealed through noise tracking.

An important task in this procedure is to determine what a "reasonable" speech level may be. Because speakers are expected to raise their vocal efforts in a noisy background, depending on the level of the background noise, it is reasonable to expect that they will be speaking at a "loud" speech or "shouted" speech level and not at a most comfortable level. Pearsons et al (1977) showed that such vocal efforts could result in speech levels ranging from $71 \mathrm{~dB}$ to $89 \mathrm{~dB}$ SPL. Consequently, it may be worthwhile to fix the speech level at $85 \mathrm{~dB}$ SPL to reflect the higher speech levels encountered in loud, noisy backgrounds.

Fixing the speech level at $85 \mathrm{~dB}$ SPL may have the following advantages. First, not all hearing aid wearers can tolerate a speech level of $85 \mathrm{~dB}$ SPL; however, the intolerance for loud sounds may be predictive of limited hearing aid use. This information would be valuable to the clinicians. Second, the resolution of the auditory system is diminished at high input levels (Wong et al, 1998; Baker and Rosen, 2002). Testing at an $85 \mathrm{~dB}$ SPL level could require examining the individual's auditory system at such levels. Because the use of hearing aids in loud, noisy situations has always been problematic (Kochkin, 2010), testing at a high $85 \mathrm{~dB}$ SPL level may be predictive of real-life hearing aid satisfaction.

These considerations prompted us to evaluate a new way of estimating noise tolerance as a means to differentiate hearing aid signal processing algorithms. We call this procedure Tracking of Noise Tolerance (TNT). This procedure estimates the SNR at which a listener is able to put up with continuous background noise while listening to speech presented at a fixed $85 \mathrm{~dB}$ SPL. Listeners are prompted to adjust the level of a continuous background noise every 3 sec while following the TNL criterion (see instructions in "Procedure" section). The average level of the noise when loudness perception has stabilized is the TNL. The difference in levels between $85 \mathrm{~dB}$ and the TNL represents the TNT index.

The purposes of this preliminary study were twofold. First, we used the TNT procedure to document how signal processing methods (amplification using wide dynamic range compression, two forms of NR, directional microphone) and hearing loss (normal versus impaired hearing) may affect the measured noise tolerance. Second, we determined the test-retest reliability of this TNT procedure.

\section{METHODS}

\section{Participants}

The data from the study by Peeters et al (2009), when subjected to a power analysis, suggested that only four participants were sufficient to reflect the statistically significant benefit of the NR algorithm at a power of 0.8 . Nonetheless, we recruited 13 hearing-impaired participants ( 9 females and 4 males) in the study. This sample size was also sufficient for the test-retest reliability measure. The average age of the participants was $65.3 \mathrm{yr}$ (standard deviation $[\mathrm{SD}]=16.8 \mathrm{yr}$; range $=$ 31-82 yr). Nine participants had prior hearing aid experience using a receiver-in-the-canal (RIC) style hearing aid, while three participants used custom hearing aids. One participant did not use any hearing aids at home, yet he participated frequently in hearing aid research studies at our facility. Excluding that last participant, the average hearing aid experience was $12.1 \mathrm{yr}(\mathrm{SD}=$ $12.8 \mathrm{yr}$ ). The mean audiogram of the hearing-impaired participants is shown in Figure 1.

Normal-hearing individuals were also included to provide a reference on how normal-hearing individuals would perform on the TNT test. Seven normal-hearing individuals (one female and six males) with thresholds $<20 \mathrm{~dB}$ HL up to $4000 \mathrm{~Hz}$ participated. Participants who exhibited up to $30 \mathrm{~dB}$ HL thresholds at 6000 and $8000 \mathrm{~Hz}$ were permitted. The average age of the normal-hearing participants was $58.1 \mathrm{yr}(\mathrm{SD}=3.7 \mathrm{yr}$; range $=50-62 \mathrm{yr}$ ). All participants signed an informed

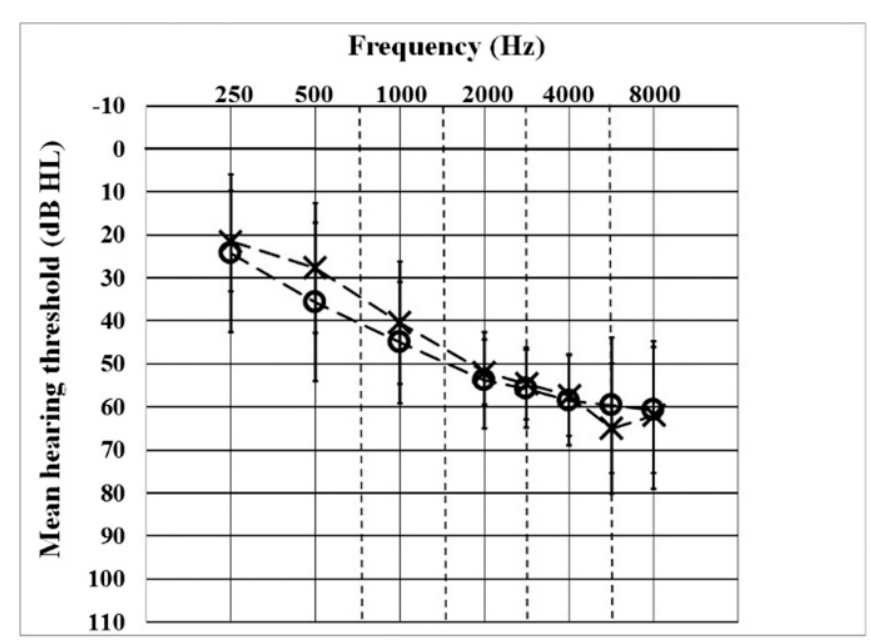

Figure 1. Average audiogram for 13 hearing-impaired participants included in the study. Error bars indicate $1 \mathrm{SD}$. 
consent upon their inclusion in the study and were financially compensated for their time.

\section{Hearing Aids}

Bilateral Widex UNIQUE Fusion 440 RIC style hearing aids were used. This is a 15-channel wide dynamic range compression hearing aid featuring a multichannel fully adaptive directional microphone, speech intelligibility-based (rtSII) NR, wind noise attenuation algorithm, active feedback cancellation, and a sound classification system. Other than the SII-based NR algorithm and the multichannel fully adaptive directional microphone, all the other features were deactivated during the study. A more detailed description of the SII-based NR algorithm and the adaptive directional microphone can be found in Peeters et al (2009). The hearing aids were coupled to the appropriate receiver ( $\mathrm{m}$ - or $\mathrm{p}$-receivers) and occluding instant-fit ear-tips. The fitting of the hearing aid followed the standard protocol of performing a feedback test, followed by measuring the in situ thresholds (Sensogram [Widex]) at $500,1000,2000$, and $4000 \mathrm{~Hz}$. The default gain settings, which were derived based on a consideration of the National Acoustics Laboratory-nonlinear version 2 rationale, were applied for all participants. All fittings were verified using simulated speech mapping measure (SoundTracker) on the Compass GPS fitting software. The sensation level shown on the SoundTracker has been demonstrated to be within $2 \mathrm{~dB}$ of real-ear measures in $84 \%$ of test participants (Oeding and Valente, 2013).

In addition, a pair of DREAM Fusion 440 RIC hearing aids was also included for evaluation. As explained in the Introduction, the DREAM was an earlier hearing aid model than the UNIQUE, with a longer NR stabilization time. Its inclusion in the study was to examine if the tracking functions from the TNT procedure could reflect such differences in processing. All adaptive features (including the directional microphone) other than the NR algorithm within the DREAM hearing aid were deactivated during the study. In addition, gain on the DREAM hearing aid was matched to the UNIQUE hearing aid across frequencies using simulated in situ frequency output curves. The participants only wore the study hearing aids in the laboratory; they did not wear the study hearing aids at home.

\section{Hearing Aid Conditions}

All hearing-impaired and normal-hearing listeners were tested in the unaided condition. All hearingimpaired listeners were also tested with the UNIQUE hearing aid and the DREAM hearing aid. The UNIQUE hearing aid was tested in the following conditions: (a) omnidirectional microphone, NR off (omni); (b) omnidirectional microphone, NR On (omni + NR); (c) directional microphone, NR Off (dir); and (d) directional microphone, NR On (dir + NR). Hearing-impaired participants were tested with the DREAM hearing aid in the following two conditions: (a) omnidirectional microphone, NR Off (omni), and (b) omnidirectional microphone, NR On (omni + NR). NR "Off" was always tested before NR "On." That is, participants tracked the TNL for 2 min in the NR "Off" before the NR was turned "On." This was done to ensure an optimal starting noise level for each individual listener during the NR "On" condition; otherwise, the variability could potentially mask the difference in stabilization times between devices. Testing order of microphone mode (directional versus omnidirectional) and hearing aids (DREAM versus UNIQUE versus unaided) was counterbalanced across participants. All participants were unaware of any test conditions (i.e., single-blinded).

\section{Stimuli}

Passages from the Connected Speech Test (CST; Cox et al, 1987) were used as stimuli. This is a story test where several related sentences on a particular topic make up a passage. Each passage contains 25 target words. These CST passages were edited to remove long pauses between sentences to create a more continuous monologue. The passages were concatenated to form new lists of related sentences, each list lasting $2 \mathrm{~min}$. These sentences were presented from a loudspeaker placed at $0^{\circ}$ azimuth at an average level of $85 \mathrm{~dB}$ SPL. A continuous speech-shaped noise available from the Hearing in Noise Test was used as the background noise. This noise is likely more sensitive to the action of an NR algorithm than that of a babble noise. Noise was presented from $90^{\circ}, 180^{\circ}$, and $270^{\circ}$ azimuths. The noise was uncorrelated by introducing a different delay across azimuths. The level of the noise was adjusted by the participants to meet the TNL criterion. The starting level of the noise was fixed at $80 \mathrm{~dB}$ SPL (i.e., $5 \mathrm{~dB}$ SNR), and a bracketing approach was used in tracking the TNL. The noise was presented simultaneously with the speech stimulus for a duration of $2 \mathrm{~min}$.

\section{Equipment and Setup}

Testing was performed in a double-walled soundtreated booth $(3 \times 3 \times 2 \mathrm{~m}$; Industrial Acoustics Company, Bronx, NY). A GSI-61 audiometer (GrasonStadler; Eden Prairie, MN) with TDH-50 (Telephonics; Farmingdale, NY) supra-aural headphones was used for audiometric measurements. A custom computer program written in Visual Basic (Microsoft; Seattle, WA) controlled the delivery of the stimuli (speech and noise), prompted the participants, and sampled the participants' TNLs. A Rotel (Tokyo, Japan) RMB-1048 amplifier and Echo Audio (Portland, OR) Gina 24 sound card were used to deliver test stimuli. Sound-field stimuli 
were presented through four KRK ST6 loudspeakers (KRK Systems, Deerfield Beach, FL; frequency response from $62 \mathrm{~Hz}$ to $20 \mathrm{kHz}, \pm 2 \mathrm{~dB}$ ) placed at $0^{\circ}, 90^{\circ}, 180^{\circ}$, and $270^{\circ}$ respectively, surrounding a chair where the participants were seated. The center of the loudspeaker driver was $107 \mathrm{~cm}$ above the floor, and the loudspeaker cones were $15 \mathrm{~cm}$ apart. A Planar 17" PT1700 MU touch screen (Leyard Company; Beijing, China) was used to collect participants' responses during testing.

The custom computer program presented uninterrupted CST sentences at $85 \mathrm{~dB}$ SPL. At the same time, the program delivered the continuous speech-shaped noise, the level of which was adjustable by the listener via the computer touch-screen monitor. The noise level was adjustable in 1-dB steps, and participants' responses were sampled every second. The program prompted the listener for a new noise level every 3 sec when no change in listeneradjusted noise level was sampled. The text prompt read, "Is the noise at a loud but tolerable level?" Participants were given three options: "try louder" to increase the level of the noise, "OK" to keep the level of the noise, or "try softer" to decrease the level of the noise. The listener may stay at the same noise level for as long as s/he deemed appropriate. The duration of the noise was fixed at 2 min for all conditions. Examination of the unaided individual tracking functions reassured us that all individual tracking stabilized to $\leq 1 \mathrm{~dB}$ in typically $<90 \mathrm{sec}$ of the tracking.

Figure 2 is an illustration of the noise tracking function during an evaluation of the NR algorithm. All tracking starts at a noise level of $80 \mathrm{~dB}$ SPL. The hearing aid will be set to NR "Off" for the first 2 min of tracking. NR will be activated for the next 2 min without the participants' knowledge. The stable noise level is recorded as L1, and is the TNL. The difference between the $85 \mathrm{~dB}$ SPL speech level and this level represents the TNT index. Thus, the lower the TNT index, the more noise the listener can tolerate. The time it takes the listener to reach a stable $\mathrm{L} 1$ level is $\mathrm{T} 1$, and it represents

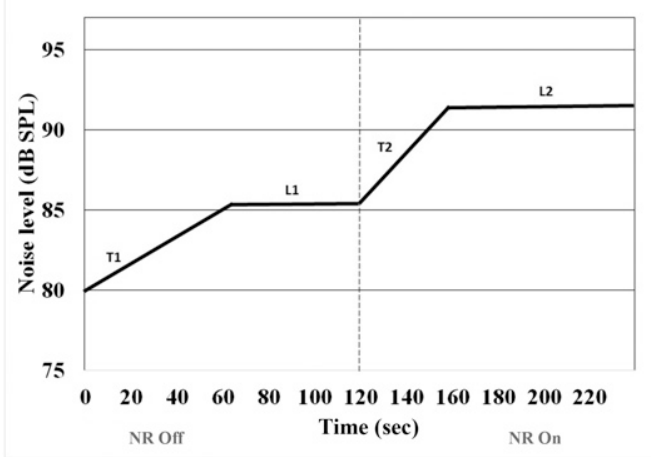

Figure 2. Hypothetical noise tracking where the first $120 \mathrm{sec}$ was evaluated without NR (NR Off) and the final 120 sec was evaluated with NR turned on (NR On). Speech was fixed at $85 \mathrm{~dB}$ SPL. NR was activated at $\mathrm{T}=121$ sec. The difference between L2 and L1 represents the benefit of the NR algorithm. the responsiveness of the participants as well as any signal processing of the hearing aid. Since the starting level is arbitrarily fixed at $80 \mathrm{~dB}$ SPL, T1 also reflects the closeness between the starting noise level and an ideal starting level. Assuming an NR algorithm improves noise tolerance, the noise level will increase from L1 to a higher level $\mathrm{L} 2$, starting at $\mathrm{T}=121 \mathrm{sec}$. The difference between $\mathrm{L} 1$ and L2 reflects the improvement in noise tolerance (or benefit) provided by the NR algorithm. The time (T2) it takes the listener to stabilize to a new noise level L2 is again determined by the listener's responsiveness and the time it takes the NR algorithm to fully stabilize. Any differences in the activation/stabilization times of an NR algorithm may result in a different T2. Because NR is activated at an ideal starting level specific to the listener (L1), T2 does not include the error incurred between using an arbitrary starting level and an ideal starting level.

\section{Procedure}

The listeners' task was to determine the unaided and aided TNL in the sound field at a fixed speech level of $85 \mathrm{~dB}$ SPL. We used the following instructions to make sure first that the speech level of $85 \mathrm{~dB}$ SPL was acceptable to the listeners:

You will be listening to a female talker reading a story at a loud volume. You should find this volume to be loud, but not uncomfortably loud. If this is not the case (i.e., too loud), please let me know and I will adjust it accordingly.

Afterward, we used the following instructions to instruct the listeners on the TNL task:

You will hear some noise in the background while you listen to the female talker. I want you to turn the noise level up as high as you can so you are no longer willing to tolerate or put up with the noise without becoming tense or tired while following the words of the story. You should bracket around that sound level to make sure that level is reached. Once you are at that level, I want you to monitor that noise level so it is at the same loudness at all times. That is, if it appears softer than before, you should turn the volume up; if it is louder than before, you should turn the volume down to keep at the same level. The level of the noise should not affect your understanding of the speech passage. If so, you should lower the loudness of the noise so speech becomes understandable again. We will prompt you periodically to remind you to keep that noise level constant.

To ensure that listeners did not tolerate more noise at the expense of speech understanding, we also estimated the speech intelligibility scores of the listeners for the CST after the tracking was completed at the stabilized noise levels when NR was "Off" and "On" (i.e., L1 and L2). A new CST passage was presented at the individual's TNT index (speech at $85 \mathrm{~dB}$ SPL and noise at 
listener's TNL). The number of words correctly repeated from the lists of sentences was recorded. Listeners who could not correctly identify $\geq 80 \%$ of the target words would be re-instructed and the TNL re-measured. Such a precaution was not needed because all listeners scored between $80 \%$ and $96 \%$ with an average of $84 \%$ at both the L1 and L2 noise levels. This assured us that the noise tolerance level was achieved with reasonably good speech understanding, and that a higher noise level (with NR activated) did not sacrifice speech understanding.

Nine of the 13 participants were able to return after 3 mo for a retest of the unaided and aided conditions with the UNIQUE hearing aid. Two runs were repeated for each listener during the second session to evaluate intrasession variability in the measured TNL. Each run was separated by $\sim 30$ min in which participants were engaged in data collection for a different study. As indicated previously, testing of microphone (omnidirectional versus directional) was counterbalanced across listeners while testing of NR "Off" always preceded NR "On."

\section{RESULTS}

$\mathrm{E}$ ach individual tracking function was analyzed to estimate the value of the stable TNL. This was achieved by examining each individual tracking function and determining when a stable judgment was reached. A straight line with a slope of " 0 " was drawn through the sampled noise levels within the steady state. The noise level at which the straight line showed the least mean deviation from the sampled noise levels was taken as the final TNL. This is equivalent to taking the averaged noise levels within the steady state. The mean TNT indices were calculated as the differences between $85 \mathrm{~dB}$ and TNL. A higher TNL represents a greater tolerance for noise, and would in turn reflect a lower TNT index. Table 1 shows the mean and SD of the TNLs and TNT indices measured across the various UNIQUE hearing aid conditions during session 1 (first) and during trials 1 and 2 in session 2. It should be noted that no retest was conducted on the normalhearing listeners.
A repeated measures analysis of variance was conducted on the hearing-impaired listeners to examine the effect of session (session 1, session 2 [test 1] and [test $2]$ ) and the effect of hearing aid condition (unaided, omni, omni + NR, dir, dir + NR). Results showed that the effect of session was not significant $\left[F_{(2,16)}=1.99\right.$, $p>0.05, \eta^{2}=0.19$, power $=0.2$ ], and the effect of hearing aid condition was significant $\left(\left[F_{(4,32)}=30.19, p<\right.\right.$ $0.001, \eta^{2}=0.79$, power $\left.=1.0\right]$. Post hoc analysis with Bonferroni adjustment indicated that the "omni" condition had significantly higher TNT indices than the four other conditions (i.e., unaided, omni + NR, dir, dir + NR). In addition, the "omni + NR" and "dir" conditions also had significantly higher TNT indices than "dir + NR" condition $(p<0.01)$.

\section{Feature Benefit}

The differences in mean TNT index (averaged across sessions and tests) between the UNIQUE hearing aid conditions were calculated to estimate the improvement in TNT index offered by each hearing aid condition. Table 2 shows the benefits (or difference in TNT indices) for each feature.

Averaged across all sessions, the use of the UNIQUE hearing aids (in an omnidirectional, no NR mode) decreased noise tolerance (increased TNT index) from the unaided condition by $\sim 4.5 \mathrm{~dB}$. The use of NR improved TNT index by $2-3 \mathrm{~dB}$, depending on the microphone used. The use of a directional microphone improved the TNT index by $3-5 \mathrm{~dB}$, depending on the NR mode. The combined use of a dir + NR improved TNT index over the Omni state by $>6.5 \mathrm{~dB}$. As indicated in Table 2, all these changes were statistically significant.

\section{NR Activation/Stabilization Times}

Figure 3 tracks the averaged TNL between the UNIQUE and the DREAM hearing aids. The TNL during first 2 min was measured without NR and the last 2 min with NR. Recall that the NR in DREAM has a longer activation and stabilization time than in

Table 1. Mean (and SD) TNL (dB SPL) and TNT index (85 - TNL, dB) Data for the Unaided, Omni, Omni + NR, Dir, and Dir + NR Conditions with the UNIQUE Hearing Aids

\begin{tabular}{|c|c|c|c|c|c|c|c|c|c|}
\hline & \multicolumn{3}{|c|}{ Session 1} & \multicolumn{6}{|c|}{ Session 2} \\
\hline & \multirow[b]{2}{*}{ TNL } & \multirow[b]{2}{*}{ TNT Index } & \multirow[b]{2}{*}{ SD } & \multicolumn{3}{|c|}{ Test 1} & \multicolumn{3}{|c|}{ Test 2} \\
\hline & & & & $\overline{T N N L}$ & TNT Index & $\overline{S D}$ & $\overline{\mathrm{TNL}}$ & TNT Index & $\overline{S D}$ \\
\hline Unaided & 87.67 & -2.67 & 3.64 & 86.33 & -1.33 & 2.92 & 88.11 & -3.11 & 2.15 \\
\hline Omni & 82.56 & 2.44 & 4.67 & 81.56 & 3.44 & 3.71 & 84.44 & 0.56 & 3.21 \\
\hline Omni + NR & 85.89 & -0.89 & 3.33 & 85.11 & -0.11 & 3.30 & 86.33 & -1.33 & 3.16 \\
\hline Dir & 87.00 & -2.00 & 5.00 & 87.00 & -2.00 & 3.24 & 87.89 & -2.89 & 2.85 \\
\hline Dir + NR & 89.67 & -4.67 & 4.61 & 88.89 & -3.89 & 2.67 & 89.56 & -4.56 & 1.88 \\
\hline Normal hearing & 90.71 & -5.71 & 1.89 & & & & & & \\
\hline
\end{tabular}

Note: Results on normal-hearing listeners are also included for comparisons (test only). 
Table 2. Improvements in TNT Index (Benefit) as a Function of Specific Hearing Aid Feature on the UNIQUE Hearing Aid

\begin{tabular}{|c|c|c|c|c|}
\hline & \multirow{2}{*}{$\frac{\text { Session } 1}{\text { TNT }}$} & \multicolumn{2}{|c|}{ Session 2} & \multirow{2}{*}{$\frac{\text { Average }}{\text { All Sessions }}$} \\
\hline & & TNT 1 & TNT 2 & \\
\hline Aided (omni-unaided) & $-5.11^{\star}$ & $-4.78^{*}$ & $-3.67^{\star}$ & $-4.52^{*}$ \\
\hline \multicolumn{5}{|l|}{ NR } \\
\hline Omni microphone & $3.33^{*}$ & $3.56^{*}$ & 1.89 & $2.93^{*}$ \\
\hline Directional microphone & $2.67^{\star}$ & $1.89^{*}$ & 1.67 & $2.07^{\star}$ \\
\hline \multicolumn{5}{|l|}{ Directional } \\
\hline No NR & $4.44^{\star}$ & $5.44^{*}$ & $3.44^{*}$ & $4.44^{*}$ \\
\hline With NR & $3.78^{*}$ & $3.78^{*}$ & $3.22^{\star}$ & $3.59^{*}$ \\
\hline $\mathrm{NR}+\mathrm{dir}$ & $7.11^{*}$ & $7.33^{*}$ & $5.11^{*}$ & $6.52^{*}$ \\
\hline
\end{tabular}

Notes: Positive number suggests an increase in noise tolerance, whereas a negative number suggests a decrease in noise tolerance. The aided benefit was taken as the difference in TNT between unaided and aided using an Omni. NR benefit was the difference in TNT between NR On and NR Off when the microphone was in an omnidirectional mode and directional mode. Directional mic benefit was the difference between directional mic and omnidirectional mic in both the NR On and Off modes. NR + dir benefit was the difference between NR On with directional mic and NR Off with omni directional mic.

*Significantly different at $p<0.01$.

the UNIQUE. The dotted curve was the mean TNL measured with the DREAM, while the solid curve was that of the UNIQUE. During the first 2 min where NR was not activated, no difference in the mean TNL was observed. Both hearing aids tracked at $\sim 84 \mathrm{~dB}$ SPL. Upon activation of the NR, the final TNL was again similar between the UNIQUE and the DREAM. The difference between the two aids was the time required to achieve the stable noise level upon activation of NR. The UNIQUE stabilized in $\leq 25$ sec after activation of the NR, while the DREAM required nearly $50 \mathrm{sec}$ to stabilize. One should remember that the listener's own reaction time was included in this time measure.

\section{Comparison to Normal-Hearing Listeners}

Table 1 also included the TNT index of normal-hearing listeners. The mean TNT index of $-5.71 \mathrm{~dB}$ was about $3 \mathrm{~dB}$ lower than the average TNT index measured with the hearing-impaired listeners in the unaided condition. Such differences narrowed to within $1 \mathrm{~dB}$ in the aided condition when both the directional microphone

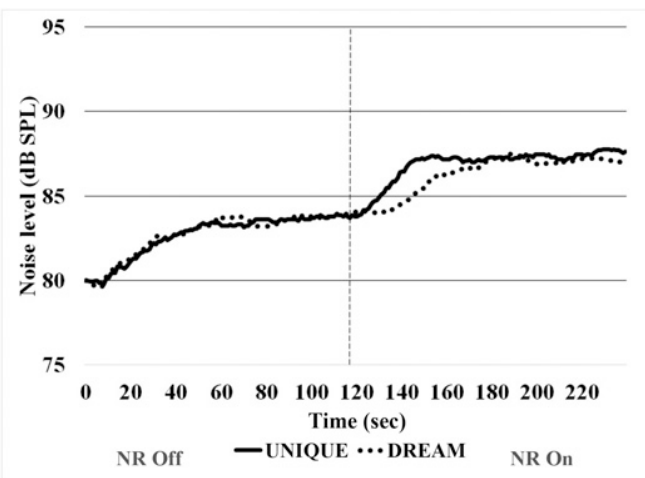

Figure 3. Averaged TNL tracking curve for UNIQUE (solid) and DREAM (dotted) hearing aids $(\mathrm{n}=13)$. and NR algorithms in the UNIQUE hearing aid were activated. A univariate analysis of variance was conducted on the TNT indices obtained at different test conditions (unaided, omni, omni $+\mathrm{NR}$, dir, and dir $+\mathrm{NR}$ ) for the hearing-impaired listeners and the unaided condition for normal-hearing listeners. Results showed that test condition was significant $\left[F_{(5,52)}=4.26\right.$, $p=0.002, \eta^{2}=0.32$, power $\left.=0.9\right]$. Post hoc analysis with Bonferroni adjustment indicated that the TNT indices obtained with normal-hearing listeners were significantly lower than the TNT indices obtained with hearing-impaired listeners aided with omnidirectional microphones, $p<0.01$. There was no significant difference in TNT indices between normal-hearing and hearing-impaired listeners in the unaided, aided with omni $+\mathrm{NR}$, dir, and dir + NR conditions, $p>0.01$.

\section{Test-Retest Reliability}

Several observations are made from Table 1 also. First, the mean TNT index measured during session 1 was similar $(<1.5 \mathrm{~dB})$ to the mean TNT index measured during the first trial in session 2 . This is true across most of the hearing aid conditions. Second, the mean TNT index measured during trial 2 in the second session was lower than that measured during the first trial (between 0.6 and $2.9 \mathrm{~dB}$ ). This suggests some learning effect when the same test was presented within the same session.

We calculated the test-retest difference in TNT index for each individual listener across all the hearing aid conditions. Intrasession variability was estimated by determining the difference in TNT index between trials 1 and 2 ( 1 versus 2 ) measured during the second session. Intersession variability was estimated by taking the difference in TNT between trial 1 in session 2 and session 1 ( 1 versus first), trial 2 in session 2 and session 1 ( 2 versus first), and the average of trials 1 and 2 in 
session 2 and session 1 (1, 2 versus first). The mean difference in TNT index measured between trials/sessions along with the standard error (se) of the measurements were then calculated. This allowed us to estimate the 95\% confidence interval (CI) by using the equation $(\mathrm{CI}=$ mean $\pm 1.96 \mathrm{se})$. Table 3 summarizes the mean, se, the range of individual differences, the $95 \% \mathrm{CI}$, as well as the upper and lower limits of the $95 \%$ CI.

Table 3 shows that the average difference in TNT index between trials was generally $<2 \mathrm{~dB}$ and fluctuated somewhat across different hearing aid conditions. As expected, the intrasession variability was smaller than the intersession variability. The 95\% CI measured within session (comparing 1 versus 2 ) varied from $1.6 \mathrm{~dB}$ (in the omni + NR mode) to $2.6 \mathrm{~dB}$ (in the dir + NR mode). Averaged across hearing aid conditions, the intrasession CI was calculated to be $2.2 \mathrm{~dB}$. The $95 \%$ CI measured between sessions (intersession variability) ranged between 3.3 and $5.6 \mathrm{~dB}$ with a mean of $4.2 \mathrm{~dB}$.

\section{DISCUSSION}

$\mathrm{T}$ he current study showed that the TNT index measured using the current protocol was $-5.7 \mathrm{~dB}$ $(\mathrm{TNL}=90.7 \mathrm{~dB}$ SPL) for normal-hearing listeners and $-2.3 \mathrm{~dB}$ for hearing-impaired listeners measured in the unaided condition (TNL $=87.3 \mathrm{~dB}$ SPL, averaged among sessions). The use of a directional microphone and NR improved TNT over the omnidirectional, NR Off conditions. The combination of these two algorithms restored TNT to within $1 \mathrm{~dB}$ of normal-hearing listeners.
The TNT procedure was reliable, yielding a CI of $2.2 \mathrm{~dB}$ when measured within a session and $4.2 \mathrm{~dB}$ between sessions. Most importantly, it enabled a differentiation between two NR algorithms that differ in their activation/stabilization times.

\section{Comparison to Previous Studies}

It is difficult to compare the results of this study to other studies because of the differences in methodology and hearing aids used. A study that is the closest in design to the current study was that conducted by Peeters et al (2009). These authors used the same stimulus conditions as the current study (CST sentences, speechshaped noise, speech front/noise surround) except that they measured ANL using its instructions and procedure (of measuring individual most comfortable level and background noise level). The authors reported an ANL benefit of $2.8 \mathrm{~dB}$ from the directional microphone with the NR Off and $2.4 \mathrm{~dB}$ with the NR On. In the current study, a directional benefit of $4.4 \mathrm{~dB}$ was reported when NR was Off, and $3.6 \mathrm{~dB}$ when NR was On. The directional benefit reported in the study aid was more than $1 \mathrm{~dB}$ higher than that reported in the Peeters et al (2009) study. Such a difference likely resulted from a difference in the effective vent used between the studies. Almost half the listeners in the Peeters et al's (2009) study used an open ear-tip, whereas all the listeners in this study wore an occluding ear-tip. As Kuk et al (2004) demonstrated, a larger vent diameter could reduce the in situ directional benefit.

Table 3. Mean, SE, Range, and 95\% Cls for the Difference in TNT Index Measured in Session 1 (First) and the Two Trials (1 and 2) during the Second Session for the Various UNIQUE Hearing Aid Conditions (Unaided, Omni, Dir, Omni + NR, and Dir + NR), $n=9$

\begin{tabular}{|c|c|c|c|c|c|c|c|}
\hline & & Mean & SE & Range & Lower & Upper & $95 \% \mathrm{Cl}$ \\
\hline \multirow[t]{4}{*}{ Unaided } & 1 vs. 2 & -1.78 & 0.49 & $(-4,0)$ & -2.75 & -0.81 & 1.94 \\
\hline & 1,2 vs. first & -0.44 & 1.07 & $(-4.5,5)$ & -2.55 & 1.66 & 4.20 \\
\hline & 1 vs. first & -1.33 & 1.11 & $(-6,4)$ & -3.50 & 0.83 & 4.33 \\
\hline & 2 vs. first & 0.44 & 1.09 & $(-3,6)$ & -1.70 & 2.59 & 4.29 \\
\hline \multirow[t]{4}{*}{ Omni } & 1 vs. 2 & -2.89 & 0.65 & $(-6,0)$ & -4.17 & -1.61 & 2.57 \\
\hline & 1,2 vs. first & 0.44 & 1.24 & $(-6,7)$ & -1.99 & 2.88 & 4.86 \\
\hline & 1 vs. first & -1.00 & 1.43 & $(-8,7)$ & -3.81 & 1.81 & 5.62 \\
\hline & 2 vs. first & 1.89 & 1.11 & $(-4,7)$ & -0.29 & 4.07 & 4.36 \\
\hline \multirow[t]{4}{*}{ Dir } & 1 vs. 2 & -0.89 & 0.54 & $(-4,1)$ & -1.94 & 0.17 & 2.11 \\
\hline & 1,2 vs. first & 0.44 & 1.00 & $(-2.5,7)$ & -1.52 & 2.41 & 3.93 \\
\hline & 1 vs. first & 0.00 & 0.78 & $(-2,5)$ & -1.53 & 1.53 & 3.06 \\
\hline & 2 vs. first & 0.89 & 1.24 & $(-3,9)$ & -1.54 & 3.32 & 4.86 \\
\hline \multirow[t]{4}{*}{ Omni + NR } & 1 vs. 2 & -1.22 & 0.40 & $(-3,0)$ & -2.01 & -0.44 & 1.57 \\
\hline & 1,2 vs. first & -0.17 & 0.86 & $(-5,4.5)$ & -1.85 & 1.51 & 3.36 \\
\hline & 1 vs. first & -0.78 & 0.86 & $(-5,4)$ & -2.47 & 0.91 & 3.38 \\
\hline & 2 vs. first & 0.44 & 0.90 & $(-5,5)$ & -1.32 & 2.21 & 3.53 \\
\hline \multirow[t]{4}{*}{ Dir + NR } & 1 vs. 2 & -0.67 & 0.67 & $(-3,2)$ & -1.97 & 0.64 & 2.61 \\
\hline & 1,2 vs. first & -0.44 & 1.15 & $(-3.5,7.5)$ & -2.70 & 1.81 & 4.51 \\
\hline & 1 vs. first & -0.78 & 1.01 & $(-5,6)$ & -2.76 & 1.20 & 3.96 \\
\hline & 2 vs. first & -0.11 & 1.36 & $(-4,9)$ & -2.77 & 2.55 & 5.33 \\
\hline
\end{tabular}


In addition, Peeters et al (2009) reported that the ANL improved by $3.2 \mathrm{~dB}$ with the use of the NR algorithm when the microphone was in the omnidirectional mode and $2.9 \mathrm{~dB}$ in the directional mode. In the current study, the improvement in TNT index from NR was $2.9 \mathrm{~dB}$ when measured with the omnidirectional microphone and $2.1 \mathrm{~dB}$ in the directional mode. This is slightly smaller than that measured in the Peeters et al (2009) study. Furthermore, the improvement in TNT index from the use of dir + NR reported in the current study was $6.5 \mathrm{~dB}$ (over the omni condition). This also compares favorably with the Peeters et al (2009) result of $5.7 \mathrm{~dB}$ from the combined use of directional microphone and NR. The NR algorithm used in the current study responds much faster $(<3 \mathrm{sec})$ than the one used in Peeters et al (2009). This improved responsiveness was observed in the current study where the TNL measured with the UNIQUE stabilized sooner than the DREAM (Figure 3). The similar outcomes between the current study and the Peeters et al study (2009) suggest that the methodological differences (fixed speech, tracking noise, instruction, etc.) between these two studies did not lead to dissimilar results. Both approaches were able to demonstrate hearing aid benefits offered by NR and directional microphone features. The advantage of the TNT is the additional timing information that is not available from the ANL procedure used by Peeters et al (2009).

\section{Test-Retest Reliability}

One of the criteria for selecting a test measure is its reliability. The level of variability must be smaller than the magnitude of the benefit offered by the hearing aid features. In this study, we attempted to minimize variability by using a fixed speech level and tracked noise tolerance over a fixed duration to reduce momentary fluctuation in loudness judgments. During a retest of the same listeners, we noted that the intrasession variation in TNT index was small, with an averaged 95\% CI of $2.2 \mathrm{~dB}$ measured across the unaided and aided conditions (various microphone and NR modes). In addition, the mean intersession variation of TNT was limited to a CI of $4.2 \mathrm{~dB}$ across the various aided conditions. Considering that each tracking trial (for each condition) takes 2 min to complete, the TNT procedure would be an efficient and reliable method to measure noise tolerance.

Because the comparison of hearing aid feature benefit is conducted within a session (i.e., intrasession in nature), any difference in the measured TNT $>2.2 \mathrm{~dB}$ could be deemed significant at the 0.05 level. In that regard, one would conclude that the NR feature $(2.5 \mathrm{~dB}$ average improvement) and the directional microphone feature ( $4 \mathrm{~dB}$ average improvement) used in the UNIQUE hearing aid resulted in a significant change in the per- ceived noise tolerance. The TNT procedure is sensitive to changes resulting from NR and directional microphone algorithms.

\section{What Does the TNT Index Tell Us?}

Because one of the design elements in this study was to ensure that listeners' choice of TNT index did not sacrifice intelligibility, we tested the listeners' understanding of the CST passages at the end of the tracking to make sure that they remained similar across test conditions. All participants understood the passages with $>80 \%$ accuracy despite the increase in noise tolerance levels from the use of NR and directional microphone. This suggests that the TNT procedure was measuring noise tolerance at a constant speech understanding level. Being able to tolerate noise in a noisy background is a precondition for the wearers' continued use of their hearing aids in noise. Listeners who can tolerate more noise are likely to spend longer time in the noise environment than those who cannot tolerate as much noise. The improvement in noise tolerance (with the use of NR and directional microphone) while preserving speech intelligibility could reduce the listening effort of the hearing aid wearers in loud, noisy environments. The current study suggests that listeners may reach a state of reduced listening effort sooner with the UNIQUE hearing aids than with the DREAM hearing aids. Considering that the TNT procedure has an intrasession $95 \% \mathrm{CI}$ of $2.2 \mathrm{~dB}$, it may be useful in differentiating hearing aid features that differ in the magnitude of the output SNR and the time course reaching that output SNR.

\section{CONCLUSIONS}

$\mathrm{T}$ he current study reported a significant $2-3 \mathrm{~dB}$ change in TNT index resulting from NR algorithm and 3.6-4.4 dB change in TNT index from a directional microphone. These data support the efficacy of the said features in improving noise tolerance without degrading speech intelligibility. In addition, the TNT procedure showed intrasession CI of $2.2 \mathrm{~dB}$ and an intersession CI of $4.2 \mathrm{~dB}$ (over three mo). This suggests that it is a sensitive and reliable measure to differentiate hearing aid processing algorithms that may differ in their output SNRs. The tracking nature of the procedure would also support evaluation of algorithms that differ in the time course in reaching the final output SNR.

\section{REFERENCES}

Baker RJ, Rosen S. (2002) Auditory filter nonlinearity in mild/ moderate hearing impairment. J Acoust Soc Am 111(3): 1330-1339. 
Chung K. (2004) Challenges and recent developments in hearing aids. Part I. Speech understanding in noise, microphone technologies and noise reduction algorithms. Trends Amplif 8(3):83-124.

Cox RM, Alexander GC, Gilmore C. (1987) Development of the Connected Speech Test (CST). Ear Hear 8(5, Suppl):119S-126S.

Desjardins JL, Doherty KA. (2014) The effect of hearing aid noise reduction on listening effort in hearing-impaired adults. Ear Hear 35(6):600-610.

Kochkin S. (2010) Marketrak VIII: consumer satisfaction with hearing aids is slowly improving. Hear $J$ 63(1):19-32.

Kuk F, Keenan D, Ludvigsen C. (2004) Is real-world directional benefit predictable? Hear Rev 11(11):18-25.

Kuk F, Paludan-Muller C. (2006) Noise management algorithm may improve speech intelligibility in noise. Hear J 59(4):62-65.

Lowery KJ, Plyler PN. (2013) The effects of noise reduction technologies on the acceptance of background noise. J Am Acad Audiol 24(8):649-659.

Marcoux AM, Yathiraj A, Côté I, Logan J. (2006) The effect of a hearing aid noise reduction algorithm on the acquisition of novel speech contrasts. Int J Audiol 45(12):707-714.

Nabelek AK, Tucker FM, Letowski TR. (1991) Toleration of background noises: relationship with patterns of hearing aid use by elderly persons. J Speech Hear Res 34(3):679-685.
Ng EH, Rudner M, Lunner T, Rönnberg J. (2015) Noise reduction improves memory for target language speech in competing native but not foreign language speech. Ear Hear 36(1):82-91.

Nilsson M, Soli SD, Sullivan JA. (1994) Development of the Hearing in Noise Test for the measurement of speech reception thresholds in quiet and in noise. J Acoust Soc Am 95(2):1085-1099.

Oeding K, Valente M. (2013) Differences in sensation level between the Widex Soundtracker and two real-ear analyzers. $J$ Am Acad Audiol 24(8):660-670.

Pearsons K, Bennett R, Fidell S. (1977) Speech Levels in Various Noise Environments. Report no EPA-600/1-77-025. Washington, DC: US Environmental protection Agency.

Peeters H, Kuk F, Lau CC, Keenan D. (2009) Subjective and objective evaluation of noise management algorithms. J Am Acad Audiol 20(2):89-98.

Ricketts TA, Hornsby BW. (2005) Sound quality measures for speech in noise through a commercial hearing aid implementing digital noise reduction. $J$ Am Acad Audiol 16(5):270-277.

Wong JC, Miller RL, Calhoun BM, Sachs MB, Young ED. (1998) Effects of high sound levels on responses to the vowel /E/ in cat auditory nerve. Hear Res 123(1-2):61-77.

Wu YH, Stangl E. (2013) The effect of hearing aid signal-processing schemes on acceptable noise levels: perception and prediction. Ear Hear 34(3):333-341. 